\title{
Polynomial identities related to special Schubert varieties
}

\author{
Francesca Cioffi ${ }^{1}$. Davide Franco ${ }^{1}$. Carmine Sessa ${ }^{1}$
}

Received: 24 November 2020 / Revised: 21 January 2021 / Accepted: 23 January 2021 /

Published online: 11 March 2021

(C) The Author(s) 2021

\begin{abstract}
Let $\mathcal{S}$ be a single condition Schubert variety with an arbitrary number of strata. Recently, an explicit description of the summands involved in the decomposition theorem applied to such a variety has been obtained in a paper of the second author. Starting from this result, we provide an explicit description of the Poincaré polynomial of the intersection cohomology of $\mathcal{S}$ by means of the Poincaré polynomials of its strata, obtaining interesting polynomial identities relating Poincaré polynomials of several Grassmannians, both by a local and by a global point of view. We also present a symbolic study of a particular case of these identities.
\end{abstract}

Keywords Leray-Hirsch theorem · Derived category · Intersection cohomology · Decomposition theorem $\cdot$ Schubert varieties $\cdot$ Resolution of singularities

Mathematics Subject Classification Primary 14B05 - Secondary 14E15 - 14F05 . $14 \mathrm{~F} 43 \cdot 14 \mathrm{~F} 45 \cdot 14 \mathrm{M} 15 \cdot 32 \mathrm{~S} 20 \cdot 32 \mathrm{~S} 60 \cdot 58 \mathrm{~K} 15$.

\section{Introduction}

In the paper [10], it was shown how one can obtain suitable polynomial identities from the study of the intersection cohomology of Schubert varieties with two strata (compare with [[10], p. 115]). The aim of our work is to extend the same approach to Special Schubert varieties with an arbitrary number of strata, by showing that the

\footnotetext{
All authors are members of GNSAGA (INdAM, Italy).

Francesca Cioffi

cioffifr@unina.it

Davide Franco

davide.franco@unina.it

Carmine Sessa

carmine.sessa2@unina.it

1 Dipartimento di Matematica e Applicazioni "R. Caccioppoli”, Università degli Studi di Napoli Federico II, Via Cintia, 80126 Napoli, Italy
} 
Poincaré polynomials of their intersection cohomology naturally lead to a class of tricky polynomial identities. In the final " 1 ", we provide some of the numerical tests for the polynomial identities that we obtained in the meantime, and a symbolic study of a particular case.

The starting point of our analysis is the main result of the paper [13], which we now summarize. Let $\mathcal{S}$ be a single condition Schubert variety or special Schubert variety of dimension $n$ (see [[5], p. 328] and [[18], Example 8.4.9]). As it is well known, $\mathcal{S}$ admits two standard resolutions: a small resolution $\xi: \mathcal{D} \rightarrow \mathcal{S}$ [[18], Definition 8.4.6] and a (usually) non-small one $\pi: \widetilde{\mathcal{S}} \rightarrow \mathcal{S}$ [[20], Sect. 3.4 and Exercise 3.4.10]. We will describe both resolutions $\pi$ and $\xi$ in Sect. 2.4. By [[15], Sect. 6.2] and [[18], Theorem 8.4.7], we have

$$
I C_{\mathcal{S}}^{\bullet} \cong R \xi_{*} \mathbb{Q}_{\mathcal{D}}[n] \text { in } D_{c}^{b}(\mathcal{S})
$$

where $I C_{\mathcal{S}}^{\bullet}$ denotes the intersection cohomology complex of $\mathcal{S}$ [[12], p. 156], and $D_{c}^{b}(\mathcal{S})$ is the constructible derived category of sheaves of $\mathbb{Q}$-vector spaces on $\mathcal{S}$.

By the celebrated Decomposition theorem [2-4,22], the intersection cohomology complex of $\mathcal{S}$ is also a direct summand of $R \pi_{*} \mathbb{Q}_{\mathcal{S}}[n]$ in $D_{c}^{b}(\mathcal{S})$. Specifically, the Decomposition theorem says that there is a decomposition in $D_{c}^{b}(\mathcal{S})$ [[4], Theorem 1.6.1]

$$
R \pi_{*} \mathbb{Q}_{\mathcal{S}}[n] \cong \oplus_{i \in \mathbb{Z}^{p}} \mathcal{H}^{i}\left(R \pi_{*} \mathbb{Q}_{\widetilde{\mathcal{S}}}[n]\right)[-i],
$$

where ${ }^{p} \mathcal{H}^{i}\left(R \pi_{*} \mathbb{Q}_{\mathcal{S}}[n]\right)$ denote the perverse cohomology sheaves [[4], Sect. 1.5]. Furthermore, the perverse cohomology sheaves ${ }^{p} \mathcal{H}^{i}\left(R \pi_{*} \mathbb{Q}_{\widetilde{S}}[n]\right)$ are semisimple, i.e. direct sums of intersection cohomology complexes of semisimple local systems, supported in the smooth strata of $\mathcal{S}$.

In the paper [13], the summands involved in (2) are explicitly described. It turns out that the semisimple local systems involved in the decomposition are constant sheaves supported in the smooth strata of $\pi$. In other words, the decomposition (2) takes the following form

$$
R \pi_{*} \mathbb{Q}_{\mathcal{S}} \cong \bigoplus_{p, q} I C_{\Delta_{p}}^{\bullet}[q]^{\oplus m_{p q}}
$$

for suitable multiplicities $m_{p q} \in \mathbb{N}_{0}$ (that are computed in [[13], Theorem 3.5]) and where the strata $\Delta_{p}$ are special Schubert varieties, as well.

Following the same lines as in [[10], section 4], our main aim is to deduce some classes of polynomial identities from the isomorphism (3). Specifically, we are going to prove a class of local identities as well as a class of global identities.

Our first task is accomplished in Theorem 2. In a nutshell, the argument behind our local polynomial identity rests on the remark that each summand of (3) is a direct sum of shifted trivial local systems in $D_{c}^{b}\left(\Delta_{p}^{0}\right)$, when restricted to the smooth part $\Delta_{p}^{0}$ of each stratum $\Delta_{p}$. This fact follows by applying the Leray-Hirsch theorem (see [[23], Theorem 7.33], [[8], Lemma 2.5]) to the summands, that are described on $\Delta_{p}^{0}$ by means of suitable Grassmann fibrations. This implies that we are allowed to associate 
a Poincaré polynomial to each summand of (3), thus providing our local identity in the stratum $\Delta_{p}^{0}$ (for more details compare with Sect. 3).

As for the global polynomial identities, they are obtained in Theorem 3, which is the main result of our paper. The idea of the proof is very similar to that of $[[10]$, section 4]. Here is the claim.

Theorem For any 4 -tuple of integers $(i, j, k, l)$ such that $0<i<j \leq k<l$ and $r=k-i<l-j=c$, we have:

$$
\begin{aligned}
& \frac{P_{j} P_{l-i}}{P_{i} P_{j-i} P_{k-i} P_{l-k}}=\frac{P_{l-j} P_{k+j-i}}{P_{k-i} P_{l+i-j-k} P_{k} P_{j-i}}+ \\
& \quad+\sum_{s=1}^{\min \{k-i, k-c\}} \frac{P_{k-c} P_{l-j} P_{k+j-i-s}}{P_{s} P_{k-c-s} P_{k-i-s} P_{l+i-j-k+s} P_{k} P_{j-i-s}} t^{2 s(c-r+s)},
\end{aligned}
$$

where we assume $P_{0}=1$ and take

$$
P_{\alpha}=h_{0} \cdot \ldots \cdot h_{\alpha-1}, \forall \alpha>0 \text { and } h_{\alpha}=\sum_{i=0}^{\alpha} t^{2 \alpha}, \forall \alpha \geq 0
$$

It is worth spending a few words about its proof. From (3) we deduce an isomorphism among the $i$-th hypercohomology spaces

$$
\mathbb{H}^{i}\left(R \pi_{*} \mathbb{Q}_{\tilde{\mathcal{S}}}\right) \cong \bigoplus_{p, q} \mathbb{H}^{i}\left(I C_{\Delta_{p}}^{\bullet}[q]^{\oplus m_{p q}}\right)
$$

that leads to an equality of the corresponding Poincaré polynomials

$$
\sum_{i} t^{i} \operatorname{dim} \mathbb{H}^{i}\left(R \pi_{*} \mathbb{Q} \tilde{\mathcal{S}}\right)=\sum_{i, p, q} t^{i} \operatorname{dim} \mathbb{H}^{i}\left(I C_{\Delta_{p}}^{\bullet}[q]^{\oplus m_{p q}}\right)
$$

Again, all summands of (5) are determined by means of Leray-Hirsch theorem as Poincaré polynomials of suitable Grassmann fibrations.

We also observe that an explicit inductive algorithm for the computation of the Poincaré polynomials of the intersection cohomology of Special Schubert varieties straightforwardly follows from our results (see Corollary 1 and Remark 2). Although these Poincaré polynomials are already known, the availability of an algorithm for their computation could be the starting point for obtaining an analogous algorithm for all Schubert varieties in a future paper, being an explicit formula in this general case not known yet.

In the "Appendix", we give an example of elementary proof of the global polynomial identity of Theorem 3 in a particular case, by algebraic manipulation only, with a divide and conquer strategy. 


\section{Basic facts and notations}

\subsection{Preliminaries}

Throughout the paper, we shall work with $\mathbb{Q}$-coefficients cohomology groups; that is, for any complex variety $V$ and any integer $k, H^{k}(V)=H^{k}(V, \mathbb{Q})$. Let $D_{c}^{b}(V)$ denote the derived category of bounded constructible complexes of sheaves $\mathcal{F}^{\bullet}$ on $V$ ([[12] 12,Sects. 1.3 and 4.1], [[4], 4, Sect. 1.5]). The $\operatorname{symbol} \mathbb{H}^{k}\left(\mathcal{F}^{\bullet}\right)$ stands for the $k$-th hypercohomology group of $\mathcal{F}^{\bullet}\left(\left[[12]\right.\right.$, Defintion 2.1.4]), while $I C_{V}^{\bullet}$ represents the intersection cohomology complex of $V$ ([[12], 12, 5.4] and [[4], 12, 1.5, Sect. 2.1]). Lastly, the intersection cohomology groups of a pure $n$-dimensional complex algebraic variety $V$ are given by ([[12], Definition 5.4.3])

$$
I H^{k}(V)=I H^{k}(V, \mathbb{Q})=\mathbb{H}^{k}\left(V, I C_{V}^{\bullet}[-n]\right) .
$$

\subsection{Decomposition theorem}

The Decomposition theorem, which was proved by A. Beilinson, J. Bernstein and P. Deligne in [2], is a tool of paramount importance: most of our results descend from it directly.

Theorem 1 (Decomposition theorem, [[4], 4, Sect. (1.6.1)]) Let $f: X \rightarrow Y$ be a proper map of complex algebraic varieties. There is an isomorphism in the constructible bounded derived category $D_{c}^{b}(Y)$

$$
R f_{*} I C_{X} \cong \bigoplus_{i \in \mathbb{Z}}^{\mathfrak{p}} \mathcal{H}^{i}\left(R f_{*} I C_{X}\right)[-i]
$$

Furthermore, the perverse sheaves ${ }^{\mathfrak{p}} \mathcal{H}^{i}\left(R f_{*} I C_{X}\right)$ are semisimple; i.e., there is a decomposition into finitely many disjoint locally closed and nonsingular subvarieties $Y=\coprod S_{\beta}$ and a canonical decomposition into a direct sum of intersection complexes of semisimple local systems

$$
{ }^{\mathfrak{p}} \mathcal{H}^{i}\left(R f_{*} I C_{X}\right) \cong \bigoplus_{\beta} I C_{\overline{S_{\beta}}}\left(L_{\beta}\right) .
$$

Roughly speaking, Decomposition theorem states that, under mild hypotheses, the direct image of the intersection cohomology complex of a complex algebraic variety can be thought of as the direct sum of intermediate extensions (see [[4], Sect. 2.7]) of semisimple local systems (see [[12], Sect. 2.5]).

In the literature one can find different approaches to the Decomposition Theorem (see [2-4,22,24]), which is a very general result but also rather implicit. On the other hand, there are many special cases for which the Decomposition Theorem admits a simplified and explicit approach. One of these is the case of varieties with isolated singularities [9,11,21]. For instance, in the work [9], a simplified approach to the Decomposition Theorem for varieties with isolated singularities is developed, in 
connection with the existence of a natural Gysin morphism, as defined in [[7], Definition 2.3] (see also [6] for other applications of the Decomposition Theorem to the Noether-Lefschetz Theory).

\subsection{Grassmannians and Poincaré polynomials}

We shall denote by $\mathbb{G}_{k}\left(\mathbb{C}^{n}\right)$ the Grassmannian of $k$-vector subspaces of $\mathbb{C}^{n}$; that is, the set of all $k$-dimensional subspaces of $\mathbb{C}^{n}$. More in general, we can extend this definition by replacing $\mathbb{C}^{n}$ with any complex vector space $V$ (see [[16], 14, Sect. 6], [[14], 16, Sect. 1.5], [[20], 20, Sect. 3.1]).

Let $X$ be a topological space. The Poincaré polynomial $H_{X}$ of its cohomology and the Poincaré polynomial I $H_{X}$ of its intersection cohomology (later on, they will be simply called Poincaré polynomials) are given by

$$
H_{X}=\sum_{\alpha \in \mathbb{Z}} \operatorname{dim}_{\mathbb{Q}} H^{\alpha}(X) \cdot t^{\alpha} \quad \text { and } \quad I H_{X}=\sum_{\alpha \in \mathbb{Z}} \operatorname{dim}_{\mathbb{Q}} I H^{\alpha}(X) \cdot t^{\alpha},
$$

respectively. When $X=\mathbb{G}_{k}\left(\mathbb{C}^{l}\right)$, we have the following explicit formula of the Poincaré polynomial (see [[5], p. 328], [[10], 10, Sect. 2 (vi), (vii),(viii)])

$$
H_{\mathbb{G}_{k}\left(\mathbb{C}^{l}\right)}=\frac{P_{l}}{P_{k} P_{l-k}}
$$

where we assume $P_{0}=1$ and take

$$
P_{\alpha}=h_{0} \cdot \ldots \cdot h_{\alpha-1}, \forall \alpha>0 \text { and } h_{\alpha}=\sum_{i=0}^{\alpha} t^{2 \alpha}, \forall \alpha \geq 0
$$

\subsection{Special Schubert varieties}

In this subsection we collect some facts concerning special Schubert varieties and their resolutions. For more details and explanations we refer the reader to [[13], Sect. 2.22.6].

Let $i, j, k, l$ be integers such that

$$
0<i<k \leq j<l \text { and } r=k-i<l-j=c
$$

and fix a $j$-dimensional subspace $F \subseteq \mathbb{C}^{l}$. We are working with single condition (or special) Schubert varieties

$$
\mathcal{S}=\left\{V \in \mathbb{G}_{k}\left(\mathbb{C}^{l}\right): \operatorname{dim}(V \cap F) \geq i\right\}
$$

and we are considering the Whitney stratification

$$
\Delta_{1} \subset \ldots \subset \Delta_{r} \subset \Delta_{r+1}=\mathcal{S}
$$


where, for any $p$,

$$
\Delta_{p}=\left\{V \in \mathbb{G}_{k}\left(\mathbb{C}^{l}\right): \operatorname{dim}(V \cap F) \geq i_{p}=k-p+1\right\}
$$

is a special Schubert variety, as well, and $\Delta_{p}=\operatorname{Sing} \Delta_{p+1}$.

$$
\begin{aligned}
& \Delta_{p q}^{0} \longleftrightarrow \tilde{\Delta}_{p} \\
& \text { For any } 0<q<p \leq r+1 \text { there is a commutative diagram } \rho_{p q} \downarrow \downarrow \pi_{p} \\
& \Delta_{q}^{0} \underset{i}{\longleftrightarrow} \Delta_{p}
\end{aligned}
$$

where

$$
\begin{aligned}
\Delta_{q}^{0} & =\Delta_{q} \backslash \operatorname{Sing} \Delta_{q}=\left\{V \in \mathbb{G}_{k}\left(\mathbb{C}^{l}\right): \operatorname{dim}(V \cap F)=i_{q}\right\}, \\
\tilde{\Delta}_{p} & =\left\{(Z, V) \in \mathbb{G}_{i_{p}}(F) \times \mathbb{G}_{k}\left(\mathbb{C}^{l}\right): Z \subseteq V\right\}, \\
\Delta_{p q}^{0} & =\pi_{p}^{-1}\left(\Delta_{q}^{0}\right) \\
& =\left\{(Z, V) \in \mathbb{G}_{i_{p}}(F) \times \mathbb{G}_{k}\left(\mathbb{C}^{l}\right): Z \subseteq V \text { and } \operatorname{dim}(V \cap F)=i_{q}\right\},
\end{aligned}
$$

the map

$$
\pi_{p}:(Z, V) \in \tilde{\Delta}_{p} \mapsto V \in \Delta_{p}
$$

is a resolution of singularities, and the function

$$
\rho_{p q}:(Z, V) \in \Delta_{p q}^{0} \mapsto V \in \Delta_{q}^{0}
$$

is a fibration with fibres

$$
F_{p q}=\mathbb{G}_{i_{p}}\left(\mathbb{C}^{i_{q}}\right) .
$$

The resolutions $\pi_{p}$ are small when $k \leq c$ (see [[13], Remark 2.3]), whereas there are other small resolutions when $k>c$ (see [[13], Proof of Lemma 3.2]); namely

$\xi_{p}:(V, U) \in \mathcal{D}_{p}=\left\{(V, U) \in \mathbb{G}_{k}\left(\mathbb{C}^{l}\right) \times \mathbb{G}_{k+j-i_{p}}\left(\mathbb{C}^{l}\right): V+F \subseteq U\right\} \mapsto V \in \Delta_{p}$

\section{Local polynomial identities}

Before we give the proof of the first theorem, we shall fix some notations in order to make it more readable. For any pair of integers $(p, q)$ with $0<q<p$, we set

$$
\begin{aligned}
m_{p} & =\operatorname{dim} \Delta_{p}=(k+1-p)(j+p-k-1)+(p-1)(l-k), \\
\delta_{p q} & =\operatorname{dim} \mathbb{G}_{p-q}\left(\mathbb{C}^{k-c}\right)=(p-q)(k-c+q-p)
\end{aligned}
$$

and

$$
A_{p q}^{\alpha}=H^{\alpha}\left(F_{p q}\right), \quad F_{p q}=\mathbb{G}_{i_{p}}\left(\mathbb{C}^{i_{q}}\right)
$$




$$
\begin{aligned}
D_{p q}^{\alpha}=H^{\alpha}\left(T_{p q}\right), & T_{p q}=\mathbb{G}_{p-q}\left(\mathbb{C}^{k-c}\right), \\
B_{p q}^{\alpha}=H^{\alpha}\left(G_{p q}\right), & G_{p q}=\mathbb{G}_{p-q}\left(\mathbb{C}^{c-q+1}\right) .
\end{aligned}
$$

Theorem 2 For any pair of integers $(p, q)$ with $0<q<p$ there is a local polynomial identity

$$
\begin{aligned}
\frac{P_{k-q+1}}{P_{k-p+1} P_{p-q}} & =\sum_{\tau=q+1}^{p-1}\left(\frac{P_{k-c}}{P_{p-\tau} P_{k-c-p+\tau}} \cdot \frac{P_{c-q+1}}{P_{\tau-q} P_{c-\tau+1}} \cdot t^{2 d_{p \tau}}\right) \\
& +\frac{P_{k-c}}{P_{p-q} P_{k-c-p+q}} \cdot t^{2 d_{p q}}+\frac{P_{c-q+1}}{P_{p-q} P_{c-p+1}},
\end{aligned}
$$

where $k \in \mathbb{Z}$ is such that $0<i<k \leq j<l, c=l-j$ and $2 d_{p \tau}=m_{p}-m_{\tau}-\delta_{p \tau}$. Proof By the Decomposition theorem [[4], Theorem 1.6.1], we know that

$$
R \pi_{p *} \mathbb{Q}_{\tilde{\Delta}_{p}}\left[m_{p}\right] \cong \bigoplus_{\alpha \in \mathbb{Z}}{ }^{p} \mathcal{H}^{\alpha}\left(R \pi_{p *} \mathbb{Q}_{\tilde{\Delta}_{p}}\left[m_{p}\right]\right)[-\alpha]
$$

In [[13], Remark 3.1] it is shown how the Leray-Hirsch theorem implies that

$$
\left.p \mathcal{H}^{\alpha}\left(i^{*} R \pi_{p *} \mathbb{Q}_{\tilde{\Delta}_{p}}\right)\right|_{\Delta_{q}^{0}} \cong A_{p q}^{\alpha-m_{q}} \otimes \mathbb{Q}_{\Delta_{q}^{0}}\left[m_{q}\right]
$$

that is,

$$
\left.{ }^{p} \mathcal{H}^{\alpha}\left(i^{*} R \pi_{p *} \mathbb{Q}_{\tilde{\Delta}_{p}}\left[m_{p}\right]\right)\right|_{\Delta_{q}^{0}} \cong A_{p q}^{\alpha+m_{p}-m_{q}} \otimes \mathbb{Q}_{\Delta_{q}^{0}}\left[m_{q}\right]
$$

(for a generalization of the Leray-Hirsch theorem in a categorical framework we refer to [[23], Theorem 7.33] and [[8], Lemma 2.5]). In addition, in [[13], Theorem 3.5] it is proved that

$$
\left.\left.p \mathcal{H}^{\alpha}\left(R \pi_{p *} \mathbb{Q}_{\tilde{\Delta}_{p}}\left[m_{p}\right]\right)\right|_{\Delta_{q}^{0}} \cong \bigoplus_{\tau=0}^{p} D_{p \tau}^{\delta_{p \tau}+\alpha} \otimes R i_{p \tau *} I C_{\Delta_{\tau}}^{\bullet}\right|_{\Delta_{q}^{0}},
$$

where $i_{p \tau}: \Delta_{\tau} \hookrightarrow \Delta_{p}$ is the inclusion. By [[13], Remark 3.3], we also have

$$
\left.I C_{\Delta_{\tau}}^{\bullet}\right|_{\Delta_{q}^{0}} \cong \bigoplus_{\beta \in \mathbb{Z}} B_{\tau q}^{\beta} \otimes \mathbb{Q}_{\Delta_{q}^{0}}\left[m_{\tau}-\beta\right] \cong \bigoplus_{\beta \in \mathbb{Z}} B_{\tau q}^{\beta+m_{\tau}} \otimes \mathbb{Q}_{\Delta_{q}^{0}}[-\beta] .
$$

Combining these results, we obtain

$$
\begin{aligned}
& \bigoplus_{\alpha \in \mathbb{Z}} A_{p q}^{\alpha+m_{p}-m_{q}} \otimes \mathbb{Q}_{\Delta_{q}^{0}}\left[m_{q}-\alpha\right] \cong \\
\cong & \bigoplus_{\alpha \in \mathbb{Z}}\left(\bigoplus_{\tau=q}^{p} D_{p \tau}^{\delta_{p \tau}+\alpha} \otimes \bigoplus_{\beta \in \mathbb{Z}} B_{\tau q}^{\beta+m_{\tau}} \otimes \mathbb{Q}_{\Delta_{q}^{0}}[-\beta]\right)[-\alpha],
\end{aligned}
$$


where $\tau \in\{q, \ldots, p\}$ because $\Delta_{\tau} \backslash \Delta_{q}^{0}=\emptyset$ whenever $\tau<q$. Since the $\gamma$-th cohomology group of a topological space is trivial when $\gamma<0$, we obtain

$$
\begin{aligned}
& \bigoplus_{\alpha \geq-m_{p}} A_{p q}^{\alpha+m_{p}} \otimes \mathbb{Q}_{\Delta_{q}^{0}}[-\alpha] \cong \\
\cong & \bigoplus_{\alpha \geq-m_{p}}\left(\bigoplus_{\tau=q}^{p} D_{p \tau}^{\delta_{p \tau}+\alpha} \otimes \bigoplus_{\beta \geq-m_{p}} B_{\tau q}^{\beta+m_{\tau}} \otimes \mathbb{Q}_{\Delta_{q}^{0}}[-\beta]\right)[-\alpha] .
\end{aligned}
$$

The right-hand complex can be rewritten as follows

$$
\begin{aligned}
& \bigoplus_{\alpha \geq-m_{p}}\left(\bigoplus_{\tau=q}^{p} D_{p \tau}^{\delta_{p \tau}+\alpha} \otimes \bigoplus_{\beta \geq-m_{p}} B_{\tau q}^{\beta+m_{\tau}} \otimes \mathbb{Q}_{\Delta_{q}^{0}}[-\beta]\right)[-\alpha] \\
\cong & \bigoplus_{\alpha, \beta \geq-m_{p}}\left(\bigoplus_{\tau=q}^{p} D_{p \tau}^{\delta_{p \tau}+\alpha} \otimes B_{\tau q}^{\beta+m_{\tau}} \otimes \mathbb{Q}_{\Delta_{q}^{0}}[-\alpha-\beta]\right)
\end{aligned}
$$

and, for any $\gamma$, its $\gamma$-th term is

$$
\bigoplus_{\alpha+\beta=\gamma}\left(\bigoplus_{\tau=q}^{p} D_{p \tau}^{\delta_{p \tau}+\alpha} \otimes B_{\tau q}^{\beta+m_{\tau}} \otimes \mathbb{Q}_{\Delta_{q}^{0}}\right)
$$

The isomorphism (6) implies that the $\gamma$-th terms of those complexes are isomorphic for any $\gamma \geq-m_{p}$; i.e.

$$
A_{p q}^{\gamma+m_{p}} \otimes \mathbb{Q}_{\Delta_{q}^{0}} \cong \bigoplus_{\alpha+\beta=\gamma}\left(\bigoplus_{\tau=q}^{p} D_{p \tau}^{\delta_{p \tau}+\alpha} \otimes B_{\tau q}^{\beta+m_{\tau}} \otimes \mathbb{Q}_{\Delta_{q}^{0}}\right)
$$

We shall observe one last thing before we compute Poincaré polynomials. For any $n \in \mathbb{N}, \mathbb{G}_{0}\left(\mathbb{C}^{n}\right)=\{0\}$, as it is the space of 0 -dimensional subspaces through the origin. As a consequence,

$$
H^{k}\left(\mathbb{G}_{0}\left(\mathbb{C}^{n}\right)\right) \cong \begin{cases}\mathbb{Q} & \text { if } k=0 \\ 0 & \text { otherwhise }\end{cases}
$$

Therefore, when $\tau=p$,

$$
\delta_{p p}=0 \text { and } D_{p p}^{\alpha} \cong \begin{cases}\mathbb{Q} & \text { if } \alpha=0 \\ 0 & \text { otherwhise }\end{cases}
$$


and, consequently,

$$
\bigoplus_{\alpha+\beta=\gamma} D_{p p}^{\alpha} \otimes B_{p q}^{\beta+m_{p}} \otimes \mathbb{Q}_{\Delta_{q}^{0}} \cong B_{p q}^{\gamma+m_{p}} \otimes \mathbb{Q}_{\Delta_{q}^{0}}
$$

Similarly, when $\tau=q$, we have

$$
B_{p q}^{\beta+m_{q}} \cong \begin{cases}\mathbb{Q} & \text { if } \beta=-m_{q} \\ 0 & \text { otherwhise }\end{cases}
$$

and

$$
\bigoplus_{\alpha+\beta=\gamma} D_{p q}^{\delta_{p q}+\alpha} \otimes B_{q q}^{\beta+m_{q}} \otimes \mathbb{Q}_{\Delta_{q}^{0}} \cong D_{p q}^{\delta_{p q}+m_{q}+\gamma} \otimes \mathbb{Q}_{\Delta_{q}^{0}} .
$$

In conclusion, for any $\gamma \geq-m_{p}$, we have

$$
\begin{aligned}
A_{p q}^{\gamma+m_{p}} \otimes \mathbb{Q}_{\Delta_{q}^{0}} \cong & \bigoplus \bigoplus_{\alpha+\beta=\gamma}\left(\bigoplus_{\tau=q+1}^{p-1} D_{p \tau}^{\delta_{p \tau}+\alpha} \otimes B_{\tau q}^{\beta+m_{\tau}} \otimes \mathbb{Q}_{\Delta_{q}^{0}}\right) \oplus \\
& \oplus\left(D_{p q}^{\delta_{p q}+m_{q}+\gamma} \otimes \mathbb{Q}_{\Delta_{q}^{0}}\right) \oplus\left(B_{p q}^{\gamma+m_{p}} \otimes \mathbb{Q}_{\Delta_{q}^{0}}\right) .
\end{aligned}
$$

Let $s=\gamma+m_{p}$ and recall that $m_{p}-m_{\tau}-\delta_{p \tau}=2 d_{p \tau}$ (see [[13], 13, Sect. 2.6]).

$$
\begin{aligned}
& \bigoplus_{\alpha+\beta=\gamma}\left(\bigoplus_{\tau=q+1}^{p-1} D_{p \tau}^{\delta_{p \tau}+\alpha} \otimes B_{\tau q}^{\beta+m_{\tau}} \otimes \mathbb{Q}_{\Delta_{q}^{0}}\right) \\
& \cong \bigoplus_{\alpha^{\prime}+\beta=s}\left(\bigoplus_{\tau=q+1}^{p-1} D_{p \tau}^{\delta_{p \tau}+\alpha^{\prime}-m_{p}} \otimes B_{\tau q}^{\beta+m_{\tau}} \otimes \mathbb{Q}_{\Delta_{q}^{0}}\right) \\
& \cong \bigoplus_{\alpha^{\prime \prime}+\beta^{\prime}=s}\left(\bigoplus_{\tau=q+1}^{p-1} D_{p \tau}^{\delta_{p \tau}+\alpha^{\prime \prime}-m_{p}+m_{\tau}} \otimes B_{\tau q}^{\beta^{\prime}} \otimes \mathbb{Q}_{\Delta_{q}^{0}}\right) \\
& \cong \bigoplus_{\alpha^{\prime \prime}+\beta^{\prime}=s}\left(\bigoplus_{\tau=q+1}^{p-1} D_{p \tau}^{\alpha^{\prime \prime}-2 d_{p \tau}} \otimes B_{\tau q}^{\beta^{\prime}} \otimes \mathbb{Q}_{\Delta_{q}^{0}}\right)
\end{aligned}
$$

where we set $\alpha^{\prime}=\alpha+m_{p}$ and $\alpha^{\prime \prime}=\alpha^{\prime}-m_{\tau}, \beta^{\prime}=\beta+m_{\tau}$. Hence, we have

$$
\begin{aligned}
A_{p q}^{s} \otimes \mathbb{Q}_{\Delta_{q}^{0}} \cong & \bigoplus_{\alpha+\beta=s}\left(\bigoplus_{\tau=q+1}^{p-1} D_{p \tau}^{\alpha-2 d_{p \tau}} \otimes B_{\tau q}^{\beta} \otimes \mathbb{Q}_{\Delta_{q}^{0}}\right) \\
& \oplus\left(D_{p q}^{s-2 d_{p q}} \otimes \mathbb{Q}_{\Delta_{q}^{0}}\right) \oplus\left(B_{p q}^{s} \otimes \mathbb{Q}_{\Delta_{q}^{0}}\right)
\end{aligned}
$$


for any $s \geq 0$. At long last, if we denote by

$$
a_{p q}^{s}=\operatorname{dim} A_{p q}^{s}, \quad d_{p q}^{s}=\operatorname{dim} D_{p q}^{s}, \quad b_{p q}^{s}=\operatorname{dim} B_{p q}^{s},
$$

we obtain identities

$$
a_{p q}^{s}=\sum_{\tau=q+1}^{p-1}\left(\sum_{\alpha+\beta=s} d_{p \tau}^{\alpha-2 d_{p \tau}} \cdot b_{\tau q}^{\beta}\right)+d_{p q}^{s-2 d_{p q}}+b_{p q}^{s}
$$

If we formally multiply both sides by $t^{s}$,

$$
\begin{aligned}
a_{p q}^{s} \cdot t^{s} & =\sum_{\tau=q+1}^{p-1}\left(\sum_{\alpha+\beta=s} d_{p \tau}^{\alpha-2 d_{p \tau}} \cdot b_{\tau q}^{\beta}\right) \cdot t^{s}+d_{p q}^{s-2 d_{p q}} \cdot t^{s}+b_{p q}^{s} \cdot t^{s} \\
& =\sum_{\tau=q+1}^{p-1}\left(\sum_{\alpha+\beta=s}\left(d_{p \tau}^{\alpha-2 d_{p \tau}} \cdot t^{\alpha-2 d_{p \tau}}\right) \cdot\left(b_{\tau q}^{\beta} \cdot t^{\beta}\right)\right) \cdot t^{2 d_{p \tau}} \\
& +\left(d_{p q}^{s-2 d_{p q}} \cdot t^{s-2 d_{p q}}\right) \cdot t^{2 d_{p q}}+b_{p q}^{s} \cdot t^{s}
\end{aligned}
$$

and if we take the sum over $s \in \mathbb{Z}$

$$
\begin{aligned}
\sum_{s} a_{p q}^{s} \cdot t^{s} & =\sum_{\tau=q+1}^{p-1}\left(\sum_{\alpha}\left(d_{p \tau}^{\alpha-2 d_{p \tau}} \cdot t^{\alpha-2 d_{p \tau}}\right) \cdot \sum_{\beta}\left(b_{\tau q}^{\beta} \cdot t^{\beta}\right)\right) \cdot t^{2 d_{p \tau}} \\
& +\sum_{s}\left(d_{p q}^{s-2 d_{p q}} \cdot t^{s-2 d_{p q}}\right) \cdot t^{2 d_{p q}}+\sum_{s} b_{p q}^{s} \cdot t^{s}
\end{aligned}
$$

We are done, because, by definition of Poincaré polynomials, the above equality becomes

$$
H_{F_{p q}}=\sum_{\tau=q+1}^{p-1}\left(H_{T_{p \tau}} \cdot H_{G_{\tau q}}\right) \cdot t^{2 d_{p \tau}}+H_{T_{p q}} \cdot t^{2 d_{p q}}+H_{G_{p q}}
$$

that is,

$$
\begin{aligned}
\frac{P_{k-q+1}}{P_{k-p+1} P_{p-q}}= & \sum_{\tau=q+1}^{p-1}\left(\frac{P_{k-c}}{P_{p-\tau} P_{k-c-p+\tau}} \cdot \frac{P_{c-q+1}}{P_{\tau-q} P_{c-\tau+1}} \cdot t^{2 d_{p \tau}}\right) \\
& +\frac{P_{k-c}}{P_{p-q} P_{k-c-p+q}} \cdot t^{2 d_{p q}}+\frac{P_{c-q+1}}{P_{p-q} P_{c-p+1}} .
\end{aligned}
$$




\section{Global polynomial identities}

We shall begin by introducing some further notations:

$$
\begin{aligned}
H_{p} & =\sum_{\alpha \in \mathbb{Z}} \operatorname{dim}_{\mathbb{Q}} H^{\alpha}\left(\tilde{\Delta}_{p}\right) \cdot t^{\alpha} \\
I_{p} & =\sum_{\alpha \in \mathbb{Z}} \operatorname{dim}_{\mathbb{Q}} I H^{\alpha}\left(\Delta_{p}\right) \cdot t^{\alpha}=\sum_{\alpha \in \mathbb{Z}} \operatorname{dim}_{\mathbb{Q}} \mathbb{H}^{\alpha}\left(I C_{\Delta_{p}}^{\bullet}\left[-m_{p}\right]\right) \cdot t^{\alpha} \\
f_{p q} & =\sum_{\alpha \in \mathbb{Z}} d_{p q}^{\alpha} \cdot t^{\alpha}=\sum_{\alpha \in \mathbb{Z}} \operatorname{dim}_{\mathbb{Q}} H^{\alpha}\left(T_{p q}\right) \cdot t^{\alpha}
\end{aligned}
$$

Recall that we defined a small resolution $\xi_{p}: \mathcal{D}_{p} \rightarrow \Delta_{p}$ as

$\xi_{p}:(V, U) \in \mathcal{D}_{p}=\left\{(V, U) \in \mathbb{G}_{k}\left(\mathbb{C}^{l}\right) \times \mathbb{G}_{k+j-i_{p}}\left(\mathbb{C}^{l}\right): V+F \subseteq U\right\} \mapsto V \in \Delta_{p}$

Remark 1 The map

$$
\varphi: U \in G:=\left\{U \in \mathbb{G}_{k+j-i_{p}}\left(\mathbb{C}^{l}\right) \mid F \subseteq U\right\} \mapsto U / F \in \mathbb{G}_{k-i_{p}}\left(\mathbb{C}^{l-j}\right),
$$

provides an isomorphism between $G$ and $\mathbb{G}_{k-i_{p}}\left(\mathbb{C}^{l-j}\right)$. Therefore, we recognize $\mathcal{D}_{p}$ as the Grassmannian of subspaces of dimension $k$ of the restriction of the tautological bundle $\mathcal{T}$ over $\mathbb{G}_{k+j-i_{p}}\left(\mathbb{C}^{l}\right)$ to the subspace $G$ :

$$
\mathcal{D}_{p} \cong \mathbb{G}_{k}\left(\left.\mathcal{T}\right|_{G}\right)
$$

By the Leray-Hirsch theorem [[23], Theorem 7.33] and the Künneth formula, we have

$$
\begin{aligned}
H^{\alpha}\left(\mathcal{D}_{p}\right) & \cong \bigoplus_{\beta \in \mathbb{Z}} H^{\beta}\left(\mathbb{G}_{k-i_{p}}\left(\mathbb{C}^{l-j}\right)\right) \otimes H^{\alpha-\beta}\left(\mathbb{G}_{k}\left(\mathbb{C}^{k+j-i_{p}}\right)\right) \cong \\
& \cong H^{\alpha}\left(\mathbb{G}_{k-i_{p}}\left(\mathbb{C}^{l-j}\right) \times \mathbb{G}_{k}\left(\mathbb{C}^{k+j-i_{p}}\right)\right),
\end{aligned}
$$

(compare also with [[8], 8, Sect. 2] for a discussion of the Leray-Hirsch theorem in a context which is closely related with that considered here).

The following formula, which represents the main result of this paper, provides a strong generalization of [[10], Sect. 2, Remark 4.2].

Theorem 3 With the same notations and conditions as Theorem 2, we have

$$
\begin{aligned}
& \frac{P_{j} P_{l-i}}{P_{i} P_{j-i} P_{k-i} P_{l-k}}=\frac{P_{l-j} P_{k+j-i}}{P_{k-i} P_{l+i-j-k} P_{k} P_{j-i}}+ \\
& +\sum_{s=1}^{\min \{k-i, k-c\}} \frac{P_{k-c} P_{l-j} P_{k+j-i-s}}{P_{s} P_{k-c-s} P_{k-i-s} P_{l+i-j-k+s} P_{k} P_{j-i-s}} t^{2 s(c-r+s)} .
\end{aligned}
$$


Proof The first part of the proof is similar to that of Theorem 2. We combine the Decomposition theorem [[4], Theorem 1.6.1] with [[13], Theorem 3.5] so as to obtain

$$
R \pi_{p *} \mathbb{Q}_{\tilde{\Delta}_{p}}\left[m_{p}\right] \cong \bigoplus_{\alpha \in \mathbb{Z}}\left(\bigoplus_{q=0}^{p} D_{p q}^{\delta_{p q}+\alpha} \otimes R i_{p q *} I C_{\Delta_{q}}^{\bullet}\right)[-\alpha]
$$

that is,

$$
\begin{aligned}
R \pi_{p *} \mathbb{Q}_{\tilde{\Delta}_{p}} \cong \bigoplus_{\alpha \in \mathbb{Z}}\left(\bigoplus_{q=1}^{p-1} D_{p q}^{\delta_{p q}+\alpha} \otimes R i_{p q *} I C_{\Delta_{q}}^{\bullet}\left[-m_{p}-\alpha\right]\right) \oplus \\
\oplus \bigoplus_{\alpha \in \mathbb{Z}}\left(D_{p 0}^{\delta_{p 0}+\alpha} \otimes R i_{p 0 *} I C_{\Delta_{0}}^{\bullet}\left[-m_{p}-\alpha\right]\right) \oplus \\
\oplus \bigoplus_{\alpha \in \mathbb{Z}}\left(D_{p p}^{\delta_{p p}+\alpha} \otimes R i_{p p *} I C_{\Delta_{p}}^{\bullet}\left[-m_{p}-\alpha\right]\right) .
\end{aligned}
$$

We have already met the term $D_{p p}^{\delta_{p p}+\alpha}$ and the second summand of the right-hand side is the zero complex since $\Delta_{0}=\emptyset$. Hence, we have

$$
\begin{aligned}
R \pi_{p *} \mathbb{Q}_{\tilde{\Delta}_{p}} & \cong \bigoplus_{\alpha \in \mathbb{Z}}\left(\bigoplus_{q=1}^{p-1} D_{p q}^{\delta_{p q}+\alpha} \otimes R i_{p q *} I C_{\Delta_{q}}^{\bullet}\left[-m_{p}-\alpha\right]\right) \oplus I C_{\Delta_{p}}^{\bullet}\left[-m_{p}\right] \\
& =\bigoplus_{\alpha \in \mathbb{Z}}\left(\bigoplus_{q=1}^{p-1} D_{p q}^{\alpha-2 d_{p q}} \otimes R i_{p q *} I C_{\Delta_{q}}^{\bullet}\left[-m_{q}-\alpha\right]\right) \oplus I C_{\Delta_{p}}^{\bullet}\left[-m_{p}\right]
\end{aligned}
$$

where we have also taken account of $m_{p}-m_{r}-\delta_{p r}=2 d_{p r}$ (see [[13],13, Sect. 2.6]).

If we apply hypercohomology, we obtain (for any $\beta \in \mathbb{Z}$ )

$$
\begin{aligned}
\mathbb{H}^{\beta}\left(R \pi_{p *} \mathbb{Q}_{\tilde{\Delta}_{p}}\right) & \cong \bigoplus_{\alpha \in \mathbb{Z}}\left(\bigoplus_{q=1}^{p-1} D_{p q}^{\alpha-2 d_{p q}} \otimes \mathbb{H}^{\beta-\alpha}\left(R i_{p q *} I C_{\Delta_{q}}^{\bullet}\left[-m_{q}\right]\right)\right) \\
& \oplus \mathbb{H}^{\beta}\left(I C_{\Delta_{p}}^{\bullet}\left[-m_{p}\right]\right)
\end{aligned}
$$

By [[12] ,Definition 5.4.3],

$$
I H^{\beta}\left(\Delta_{p}\right)=\mathbb{H}^{\beta}\left(I C_{\Delta_{p}}^{\bullet}\left[-m_{p}\right]\right)
$$


and, by [[12], Definition 2.1.4] and [[17], Chapter II, (4.5)],

$$
\begin{aligned}
\mathbb{H}^{\beta}\left(R i_{p q *} I C_{\Delta_{q}}^{\bullet}\right) & =H^{\beta}\left(\Gamma\left(\Delta_{p}, i_{p q *} I^{\bullet}\right)\right)=H^{\beta}\left(\Gamma\left(i_{p q *}^{-1}\left(\Delta_{p}\right), I^{\bullet}\right)\right)= \\
& =H^{\beta}\left(\Gamma\left(\Delta_{q}, I^{\bullet}\right)\right)=\mathbb{H}^{\beta}\left(I C_{\Delta_{q}}^{\bullet},\right.
\end{aligned}
$$

where $I C_{\Delta_{q}}^{\bullet} \rightarrow I^{\bullet}$ is an injective resolution of $I C_{\Delta_{q}}^{\bullet}$ and $\Gamma$ is the global section functor. Similarly,

$$
\begin{aligned}
\mathbb{H}^{\beta}\left(R \pi_{p *} \mathbb{Q}_{\tilde{\Delta}_{p}}\right) & =H^{\beta}\left(\Gamma\left(\Delta_{p}, R \pi_{p *} \mathbb{Q}_{\tilde{\Delta}_{p}}\right)\right)=H^{\beta}\left(\Gamma\left(\Delta_{p}, \pi_{p *} I^{\bullet}\right)\right) \\
& =H^{\beta}\left(\Gamma\left(\pi_{p *}^{-1}\left(\Delta_{p}\right), I^{\bullet}\right)\right)=H^{\beta}\left(\Gamma\left(\tilde{\Delta}_{p}, I^{\bullet}\right)\right) \\
& =H^{\beta}\left(\tilde{\Delta}_{p}, \mathbb{Q}_{\tilde{\Delta}_{p}}\right)=H^{\beta}\left(\tilde{\Delta}_{p}\right)
\end{aligned}
$$

where $\mathbb{Q}_{\tilde{\Delta}_{p}} \rightarrow I^{\bullet}$ is an injective resolution and the last equality is [[17], Theorem 7.12, p. 242].

Substituting in the above isomorphism, we obtain

$$
H^{\beta}\left(\tilde{\Delta}_{p}\right) \cong \bigoplus_{\alpha \in \mathbb{Z}}\left(\bigoplus_{q=1}^{p-1} D_{p q}^{\alpha-2 d_{p q}} \otimes I H^{\beta-\alpha}\left(\Delta_{q}\right)\right) \oplus \mathbb{H}^{\beta}\left(I C_{\Delta_{p}}^{\bullet}\left[-m_{p}\right]\right)
$$

As we did in the proof of Theorem 2, we conclude

$$
\begin{aligned}
\sum_{\beta \in \mathbb{Z}} \operatorname{dim} H^{\beta}\left(\tilde{\Delta}_{p}\right) \cdot t^{\beta} & =\sum_{q=1}^{p-1} \sum_{\alpha, \beta \in \mathbb{Z}} d_{p q}^{\alpha-2 d_{p q}} \cdot t^{\alpha-2 d_{p q}} . \\
& \cdot \operatorname{dim} I H^{\beta-\alpha}\left(\Delta_{q}\right) \cdot t^{\beta-\alpha} \cdot t^{2 d_{p q}}+\sum_{\beta \in \mathbb{Z}} \operatorname{dim} I H^{\beta}\left(\Delta_{p}\right) \cdot t^{\beta},
\end{aligned}
$$

which can be compactly rewritten as

$$
H_{p}=I_{p}+\sum_{q=1}^{p-1} f_{p q} \cdot I_{q} \cdot t^{2 d_{p q}}
$$

Again, Leray-Hirsch theorem implies that $\tilde{\Delta}_{p}$ has the same Poincaré polynomial as $\mathbb{G}_{i_{p}}(F) \times \mathbb{G}_{k-i_{p}}\left(\mathbb{C}^{l-i_{p}}\right)$. Thus, the left-hand side is

$$
H_{p}=H_{\mathbb{G}_{i p}(F) \times \mathbb{G}_{k-i p}\left(\mathbb{C}^{l-i_{p}}\right)}=H_{\mathbb{G}_{i_{p}}(F)} \cdot H_{\mathbb{G}_{k-i p}\left(\mathbb{C}^{l-i_{p}}\right)} \cdot
$$

By virtue of [[13], Formula (19)] and Remark 1, we have

$$
I H^{\alpha}\left(\Delta_{p}\right)=\mathbb{H}^{\alpha}\left(\Delta_{p}, I C_{\Delta_{p}}^{\bullet}\left[-m_{p}\right]\right)=\mathbb{H}^{\alpha}\left(\Delta_{p}, R \xi_{p *} \mathbb{Q}_{\mathcal{D}_{p}}\right)
$$




$$
=H^{\alpha}\left(\mathcal{D}_{p}\right) \cong \bigoplus_{\beta \in \mathbb{Z}} H^{\beta}\left(\mathbb{G}_{k-i_{p}}\left(\mathbb{C}^{l-j}\right)\right) \otimes H^{\alpha-\beta}\left(\mathbb{G}_{k}\left(\mathbb{C}^{k+j-i_{p}}\right)\right)
$$

in other words,

$$
I_{p}=H_{\mathbb{G}_{k-i p}}\left(\mathbb{C}^{l-j}\right) \times \mathbb{G}_{k}\left(\mathbb{C}^{k+j-i p}\right)=H_{\mathbb{G}_{k-i} p}\left(\mathbb{C}^{l-j}\right) \cdot H_{\mathbb{G}_{k}\left(\mathbb{C}^{k+j-i_{p}}\right)}
$$

Adopting the same notations as $§ 2.3$, we have

$$
\begin{aligned}
H_{p} & =\frac{P_{j}}{P_{i_{p}} P_{j-i_{p}}} \cdot \frac{P_{l-i_{p}}}{P_{k-i_{p}} P_{l-k}}, \\
I_{p} & =\frac{P_{l-j}}{P_{k-i_{p}} P_{l-j-k+i_{p}}} \cdot \frac{P_{k+j-i_{p}}}{P_{k} P_{j-i_{p}}}
\end{aligned}
$$

and

$$
f_{p q}=\frac{P_{k-c}}{P_{p-q} P_{k-c-(p-q)}} .
$$

Formula (7) becomes

$$
\begin{gathered}
\frac{P_{j} P_{l-i_{p}}}{P_{i_{p}} P_{j-i_{p}} P_{k-i_{p}} P_{l-k}}=\frac{P_{l-j} P_{k+j-i_{p}}}{P_{k-i_{p}} P_{l-j-k+i_{p}} P_{k} P_{j-i_{p}}}+ \\
+\sum_{q=1}^{\min \{p-1, k-c-p\}} \frac{P_{k-c} P_{l-j} P_{k+j-i_{q}}}{P_{p-q} P_{k-c-(p-q)} P_{k-i_{q}} P_{l-j-k+i_{q}} P_{k} P_{j-i_{q}}} t^{2 d_{p q}} .
\end{gathered}
$$

Since we are interested in studying the Poincaré polynomials of the Schubert variety $\mathcal{S}$, we are going to take $p=r+1$. Bearing in mind that $i_{q}=k-q+1$ (in particular, $i_{p}=i_{r+1}=k-r=i$ ) and $c=l-j$, if we set $s=p-q=r+1-q$, we have (from left to right, numerators first)

$$
\begin{aligned}
l-i_{p} & =l-i, \\
k+j-i_{p} & =k+j-i, \\
k+j-i_{q} & =j+q-1=j+r-s=j+k-i-s, \\
j-i_{p} & =j-i \\
k-i_{p} & =k-i \\
l-j-k+i_{p} & =l+i-j-k, \\
k-i_{q} & =q-1=r-s=k-i-s, \\
l-j-k+i_{q} & =l-j-q+1=l-j+s-r=l-j+s-k+i \\
j-i_{q} & =j-k+q-1=j-k+r-s=j-i-s
\end{aligned}
$$


and the previous equality becomes $\left(2 d_{p q}=2(p-q)(c+1-q)=2 s(c+s-r)\right)$

$$
\begin{aligned}
& \frac{P_{j} P_{l-i}}{P_{i} P_{j-i} P_{k-i} P_{l-k}}=\frac{P_{l-j} P_{k+j-i}}{P_{k-i} P_{l+i-j-k} P_{k} P_{j-i}}+ \\
& +\sum_{s=1}^{\min \{k-i, k-c\}} \frac{P_{k-c} P_{l-j} P_{k+j-i-s}}{P_{s} P_{k-c-s} P_{k-i-s} P_{l+i-j-k+s} P_{k} P_{j-i-s}} t^{2 s(c-r+s)} .
\end{aligned}
$$

Corollary 1 For all $p=2, \ldots, r+1$ one has:

$$
I_{p}=H_{p}-\sum_{q=1}^{p-1} P_{p q}(t)=H_{p}-\sum_{q=1}^{p-1} t^{2 d_{p q}} f_{p q} I_{q} .
$$

Remark 2 From the previous corollary we get an explicit inductive algorithm for the computation of Poincaré polynomials of the intersection cohomology of Special Schubert varieties, which is described by the following equality, where we put $g_{p q}=t^{2 d_{p q}} f_{p q}$ in order to simplify the notation:

$$
\begin{aligned}
& {\left[\begin{array}{c}
I_{r+1} \\
I_{r} \\
\cdot \\
\cdot \\
\cdot \\
I_{2} \\
I_{1}
\end{array}\right]=\left[\begin{array}{cccccc}
1 & g_{r+1, r} & g_{r+1, r-1} & g_{r+1, r-2} & \ldots & g_{r+1,1} \\
0 & 1 & g_{r, r-1} & g_{r, r-2} & \ldots & g_{r, 1} \\
0 & 0 & 1 & g_{r-1, r-2} & \ldots & g_{r-1,1} \\
\cdot & \cdot & \cdot & \cdot & \cdot & \cdot \\
\cdot & \cdot & \cdot & \cdot & \cdot & \cdot \\
0 & 0 & 0 & \ldots & 1 & g_{21} \\
0 & 0 & 0 & 0 & \ldots & 1
\end{array}\right]^{-1} \cdot\left[\begin{array}{c}
H_{r+1} \\
H_{r} \\
\cdot \\
\cdot \\
\cdot \\
H_{2} \\
H_{1}
\end{array}\right]} \\
& =\sum_{k=0}^{r}(-1)^{k}\left[\begin{array}{cccccc}
0 & g_{r+1, r} & g_{r+1, r-1} & g_{r+1, r-2} & \ldots & g_{r+1,1} \\
0 & 0 & g_{r, r-1} & g_{r, r-2} & \ldots & g_{r, 1} \\
0 & 0 & 0 & g_{r-1, r-2} & \cdots & g_{r-1,1} \\
\cdot & \cdot & . & \cdot & \cdot & \cdot \\
\cdot & \cdot & . & \cdot & \cdot & \cdot \\
0 & 0 & 0 & \ldots & 0 & g_{21} \\
0 & 0 & 0 & 0 & \ldots & 0
\end{array}\right]^{k} \cdot\left[\begin{array}{c}
H_{r+1} \\
H_{r} \\
\cdot \\
\cdot \\
\cdot \\
H_{2} \\
H_{1}
\end{array}\right] .
\end{aligned}
$$

Funding Open access funding provided by Universitá degli Studi di Napoli Federico II within the CRUICARE Agreement.

Open Access This article is licensed under a Creative Commons Attribution 4.0 International License, which permits use, sharing, adaptation, distribution and reproduction in any medium or format, as long as you give appropriate credit to the original author(s) and the source, provide a link to the Creative Commons licence, and indicate if changes were made. The images or other third party material in this article are included in the article's Creative Commons licence, unless indicated otherwise in a credit line to the material. If material is not included in the article's Creative Commons licence and your intended use is not permitted by statutory regulation or exceeds the permitted use, you will need to obtain permission directly from the copyright holder. To view a copy of this licence, visit http://creativecommons.org/licenses/by/4.0/. 


\section{Appendix: a symbolic point of view}

In this "Appendix", we consider the global polynomial identity of Theorem 3 from a symbolic point of view. Recall that the requests over the integers $i, j, k, j, l$ and the values $r:=k-i, c:=l-j$ are

$$
0<i<k \leq j<l \text { and } 0<r<c<k
$$

or, equivalently,

$$
0<i<j \text { and } 0<r<c<r+i \leq j .
$$

Nevertheless, if we also assume $P_{\alpha}=0$ for every $\alpha<0$, the polynomial identity of Theorem 3 symbolically makes sense, i.e. the denominators do not vanish, under the following weaker assumptions:

$$
0 \leq i \leq k \leq j \text { and } 0 \leq r \leq c \leq k
$$

or, equivalently,

$$
0 \leq i \leq j \text { and } 0 \leq r \leq c \leq r+i \leq j .
$$

However, in the few further cases $r=0$ or $c=r+i$ or $i=0$ or $i=j$, which we have under the assumptions (9), the polynomial identity trivially holds. For the remaining case $c=r$, we obtain some experimental evidences verifying the polynomial identity for the 4-uples $(i, j, c, r)$, where $c=r$ varies in [2, 20], $i$ in [1,20] and $j$ in $[r+i, 40]$, by direct computations performed in CoCoA5 (see [1]).

In the following, we consider the assumptions (8) (except for some special cases that will be highlighted), and give a proof of that identity by a mere algebraic manipulation when $2=\min \{k-i, k-c\}$, which is the first case with a significant geometric meaning in the context of this paper.

By direct computations, we also verified that the polynomial identity of Theorem 3 holds for all the 4-uples $(i, j, c, r)$ where $i$ varies in [1, 20], $r$ in [2, 20], $j$ in $[r+i, 40]$ and $c$ in $[r+1, r+i-1]$. Some CoCoA5 functions that perform such computations are available at http://wpage.unina.it/cioffifr/PolynomialIdentity.

\section{Case $2=k-i \leq k-c$}

With the same notation of Theorem 3 and recalling that $k=i+2$ and $\ell=j+c$, the global polynomial identity becomes:

$$
\begin{gathered}
\frac{P_{j} P_{j+c-i}}{P_{i} P_{j-i} P_{2} P_{j+c-i-2}}=\frac{P_{c} P_{j+2}}{P_{2} P_{c-2} P_{i+2} P_{j-i}}+ \\
+t^{2(c-1)} \frac{P_{i-c+2} P_{c} P_{j+1}}{P_{i-c+1} P_{c-1} P_{i+2} P_{j-i-1}}+t^{4 c} \frac{P_{i-c+2} P_{j}}{P_{2} P_{i-c} P_{i+2} P_{j-i-2}}
\end{gathered}
$$

where only the parameters $i, j, c$ appear. Note that formula (10) does make sense for every $j \geq i+2$ and $c \geq 2$. Let 


$$
\begin{aligned}
H & :=\frac{P_{j} P_{j+c-i}}{P_{i} P_{j-i} P_{2} P_{j+c-i-2}}, \quad F:=\frac{P_{c} P_{j+2}}{P_{2} P_{c-2} P_{i+2} P_{j-i}}, \\
F_{1} & :=t^{2(c-1)} \frac{P_{i-c+2} P_{c} P_{j+1}}{P_{i-c+1} P_{c-1} P_{i+2} P_{j-i-1}}, \quad F_{2}:=t^{4 c} \frac{P_{i-c+2} P_{j}}{P_{2} P_{i-c} P_{i+2} P_{j-i-2}} .
\end{aligned}
$$

Since $\frac{P_{\alpha}}{P_{\alpha-h}}=h_{\alpha-1} \ldots h_{\alpha-h}$, for every $\alpha>h \geq 0$, we compute $F:=\frac{h_{c-2} h_{c-1} P_{j+2}}{h_{1} P_{i+2} P_{j-i}}$ and observe that

$$
\begin{aligned}
& H=F\left[\frac{h_{j+c-i-2} h_{j+c-i-1} h_{i} h_{i+1}}{h_{j} h_{j+1} h_{c-2} h_{c-1}}\right], F_{1}=F\left[t^{2(c-1)} \frac{h_{1} h_{i-c+1} h_{j-i-1}}{h_{j+1} h_{c-2}}\right], \\
& F_{2}=F\left[t^{4 c} \frac{h_{i-c} h_{i-c+1} h_{j-i-2} h_{j-i-1}}{h_{c-2} h_{c-1} h_{j} h_{j+1}}\right] .
\end{aligned}
$$

Hence, letting

$$
\begin{aligned}
F(i, j, c) & :=\frac{h_{j+c-i-2} h_{j+c-i-1} h_{i} h_{i+1}}{h_{j} h_{j+1} h_{c-2} h_{c-1}}+ \\
& -t^{2(c-1)} \frac{h_{1} h_{i-c+1} h_{j-i-1}}{h_{j+1} h_{c-2}}-t^{4 c} \frac{h_{i-c} h_{i-c+1} h_{j-i-2} h_{j-i-1}}{h_{c-2} h_{c-1} h_{j} h_{j+1}},
\end{aligned}
$$

formula (10) holds if and only if $F(i, j, c)=1$. Observe that $F(i, j, c)$ does make sense for every $c \geq 2$ and for every positive integers $i, j$. In the following, we repeatedly apply the equality $t^{2 \alpha} h_{\beta}=h_{\alpha+\beta}-h_{\alpha-1}$, which holds for every $\alpha, \beta \geq 0$.

We now show that $F(i, j, 3)=1$ for $j \geq i$, where

$$
F(i, j, 3)=\frac{h_{i} h_{i+1}}{h_{j} h_{j+1}} \frac{h_{j-i+1} h_{j-i+2}}{P_{3}}-t^{4} \frac{h_{i-2} h_{j-i-1}}{h_{j+1}}-t^{12} \frac{h_{j-i-2} h_{j-i-1} h_{i-2} h_{i-3}}{P_{3} h_{j} h_{j+1}} .
$$

Although we should consider $i \geq c \geq 3$ due to (8), symbolically we easily obtain

$$
\begin{array}{r}
F(2, j, 3)=\frac{h_{2} h_{3}}{h_{j} h_{j+1}} \frac{h_{j} h_{j-1}}{h_{1} h_{2}}-t^{4} \frac{h_{j-3} h_{1}}{h_{j+1} h_{1}}=\frac{h_{3}}{h_{j+1}} \frac{h_{j-1}}{h_{1}}-\frac{\left(h_{j-1}-h_{1}\right) h_{1}}{h_{1} h_{j+1}}= \\
\frac{\left(h_{3}-h_{1}\right) h_{j-1}+h_{1}^{2}}{h_{1} h_{j+1}}=\frac{t^{4}\left(1+t^{2}\right) h_{j-1}+h_{1}^{2}}{h_{1} h_{j+1}}=\frac{h_{j+1}-h_{1}+h_{1}}{h_{j+1}}=1 .
\end{array}
$$

Hence, by induction, we can assume $F(i-1, j, 3)=1$ and prove that $F(i, j, 3)-$ $F(i-1, j, 3)$ is null, for every $i>2$. We compute

$$
\begin{array}{r}
F(i, j, 3)-F(i-1, j, 3)=\frac{h_{i} h_{j-i+2}\left(h_{i+1} h_{j-i+1}-h_{i-1} h_{j-i+3}\right)}{h_{j} h_{j+1} P_{3}}+ \\
-\frac{t^{4} h_{j} P_{3}\left(h_{i-2} h_{j-i-1}-h_{i-3} h_{j-i}\right)}{h_{j} h_{j+1} P_{3}}-\frac{t^{12} h_{j-i-1} h_{i-3}\left(h_{j-i-2} h_{i-2}-h_{j-i} h_{i-4}\right)}{h_{j} h_{j+1} P_{3}} .
\end{array}
$$

So, letting $G_{0}:=h_{i} h_{j-i+2}\left(h_{i+1} h_{j-i+1}-h_{i-1} h_{j-i+3}\right)$

$$
\begin{aligned}
& G_{1}:=-t^{4} h_{j} P_{3}\left(h_{i-2} h_{j-i-1}-h_{i-3} h_{j-i}\right) \\
& G_{2}:=-t^{12} h_{j-i-1} h_{i-3}\left(h_{j-i-2} h_{i-2}-h_{j-i} h_{i-4}\right),
\end{aligned}
$$


we obtain $F(i, j, 3)-F(i-1, j, 3)=0$ if and only if $G_{0}+G_{1}+G_{2}=0$.

Being $h_{i+1}=t^{4} h_{i-1}+h_{1}$ e $h_{j-i+3}=t^{4} h_{j-i+1}+h_{1}, G_{0}$ becomes:

- $G_{0}=h_{i} h_{j-i+2} h_{1}\left(h_{j-i+1}-h_{i-1}\right)$.

Being $h_{i-2}=t^{2} h_{i-3}+h_{0}, h_{j-i}=t^{2} h_{j-i-1}+h_{0}$ and then $t^{4} h_{i-3}=h_{i-1}-h_{1}$, $t^{4} h_{j-i-1}=h_{j-i+1}-h_{1}, G_{1}$ becomes:

$-G_{1}=h_{j} P_{3}\left(h_{i-1}-h_{j-i+1}\right)$.

Being $h_{j-i}=t^{4} h_{j-i-2}+h_{1}$ e $h_{i-2}=t^{4} h_{i-4}+h_{1}, G_{2}$ becomes

$-G_{2}=t^{6} h_{j-i-1} h_{i-3} h_{1}\left(h_{i-1}-h_{j-i+1}\right)$.

So, we obtain $G_{0}+G_{1}+G_{2}=h_{1}\left(h_{i-1}-h_{j-i+1}\right)\left(h_{i} h_{j-i+2}-h_{j} h_{2}-\right.$ $\left.t^{6} h_{j-i+1} h_{i-3}\right)=0$.

Recall that we are assuming $k-i \leq k-c$, hence $i \geq c$, and we know that $F(i, j, 3)=1$. So, our thesis now is $F(i, j, c)-F(i, j, c-1)=0$. Assuming $c>3$, we compute

$$
\begin{aligned}
F(i, j, c)-F(i, j, c-1)= & \frac{h_{j+c-i-2} h_{j+c-i-1} h_{i} h_{i+1}}{h_{j} h_{j+1} h_{c-2} h_{c-1}}-\frac{h_{j+c-i-3} h_{j+c-i-2} h_{i} h_{i+1}}{h_{j} h_{j+1} h_{c-3} h_{c-2}}+ \\
& -t^{2(c-1)} \frac{h_{1} h_{i-c+1} h_{j-i-1}}{h_{j+1} h_{c-2}}+t^{2(c-2)} \frac{h_{1} h_{i-c+2} h_{j-i-1}}{h_{j+1} h_{c-3}}+ \\
& -t^{4 c} \frac{h_{i-c} h_{i-c+1} h_{j-i-2} h_{j-i-1}}{h_{c-2} h_{c-1} h_{j} h_{j+1}}+t^{4(c-1)} \frac{h_{i-c+1} h_{i-c+2} h_{j-i-2} h_{j-i-1}}{h_{c-3} h_{c-2} h_{j} h_{j+1}} .
\end{aligned}
$$

So, letting

$$
\begin{aligned}
& Q_{0}:=h_{j+c-i-2} h_{j+c-i-1} h_{i} h_{i+1} h_{c-3}-h_{j+c-i-3} h_{j+c-i-2} h_{i} h_{i+1} h_{c-1} \\
& Q_{1}:=-t^{2(c-1)} h_{1} h_{i-c+1} h_{j-i-1} h_{j} h_{c-1} h_{c-3}+t^{2(c-2)} h_{1} h_{i-c+2} h_{j-i-1} h_{j} h_{c-1} h_{c-2} \\
& Q_{2}:=-t^{4 c} h_{i-c} h_{i-c+1} h_{j-i-2} h_{j-i-1} h_{c-3}+t^{4(c-1)} h_{i-c+1} h_{i-c+2} h_{j-i-2} h_{j-i-1} h_{c-1}, \\
& \text { we have } F(i, j, c)-F(i, j, c-1)=0 \text { if and only if } Q_{0}+Q_{1}+Q_{2}=0 .
\end{aligned}
$$

Being $h_{j+c-i-1}=t^{4} h_{j+c-i-3}+h_{1}$ and $t^{4} h_{c-3}=h_{c-1}-h_{1}$, then $Q_{0}$ becomes

$$
\text { - } Q_{0}=h_{j+c-i-2} h_{1} h_{i} h_{i+1}\left(h_{c-3}-h_{j+c-i-3}\right)=-t^{2(c-2)} h_{j+c-i-2} h_{1} h_{i} h_{i+1} h_{j-i-1} \text {. }
$$

Being $t^{2(c-1)} h_{i-c+1}=h_{i}-h_{c-2}$ and $t^{2(c-2)} h_{i-c+2}=h_{i}-h_{c-3}$, then $Q_{1}$ becomes

$$
\text { - } Q_{1}=t^{2(c-2)} h_{1} h_{i} h_{j} h_{c-1} h_{j-i-1} \text {. }
$$

Being $t^{4} h_{i-c}=h_{i-c+2}-h_{1}$, then $Q_{2}$ becomes

- $Q_{2}=t^{4(c-1)} h_{i-c+1} h_{j-i-2} h_{j-i-1}\left(\left(h_{i-c+2}-h_{1}\right) h_{c-3}+h_{i-c+2} h_{c-1}\right)$.

Note that, if $j=i$ or $j=i+1$, then $Q_{0}+Q_{1}=0=Q_{2}$. So, we now consider the less obvious case $j>i+1$. Observe that

$$
\begin{aligned}
Q_{1}+Q_{0}= & t^{2(c-2)} h_{1} h_{i} h_{j-i-1}\left(h_{j} h_{c-1}-h_{i+1} h_{j+c-i-2}\right)= \\
& t^{2(c-2)} h_{1} h_{i} h_{j-i-1} h_{j-i-2}\left(h_{c-1}-h_{i+1}\right)=-t^{2(c-2)} h_{1} h_{i} h_{j-i-1} h_{j-i-2} t^{2 c} h_{i-c+1} .
\end{aligned}
$$

We are done, because: 


$$
\begin{aligned}
Q_{1}+Q_{0}+Q_{2} & =t^{4(c-1)} h_{i-c+1} h_{j-i-2} h_{j-i-1}\left(-h_{1} h_{i}+\left(h_{i-c+2}-h_{1}\right) h_{c-3}+h_{i-c+2} h_{c-1}\right)= \\
& =t^{4(c-1)} h_{i-c+1} h_{j-i-2} h_{j-i-1} h_{i-c+2}\left(t^{2(c-1)}+t^{2(c-2)}-h_{1} t^{2(c-2)}\right)=0 .
\end{aligned}
$$

\section{Case $2=k-c<k-i$}

In this case we have $r+i=k=c+2, \ell=j+c, c=r+i-2$, and the global polynomial identity becomes:

$$
\begin{gathered}
\frac{P_{j} P_{j+r-2}}{P_{i} P_{j-i} P_{r} P_{j-2}}=\frac{P_{r+i-2}}{P_{r} P_{i-2}} \frac{P_{r+j}}{P_{r+i} P_{j-i}}+ \\
+t^{2(i-1)} \frac{P_{2} P_{r+i-2} P_{r+j-1}}{P_{r-1} P_{i-1} P_{r+i} P_{j-i-1}}+t^{4 i} \frac{P_{2} P_{r+i-2} P_{r+j-2}}{P_{2} P_{r-2} P_{i} P_{r+i} P_{j-i-2}}
\end{gathered}
$$

where only the parameters $i, j, r$ appear. Note that formula (11) does make sense for every $2 \leq r$ and $2 \leq i \leq j-2$. Let

$$
\begin{aligned}
K & :=\frac{P_{j} P_{j+r-2}}{P_{i} P_{j-i} P_{r} P_{j-2}}, E:=\frac{P_{r+i-2}}{P_{r} P_{i-2}} \frac{P_{r+j}}{P_{r+i} P_{j-i}}, \\
E_{1} & :=t^{2(i-1)} \frac{P_{2} P_{r+i-2} P_{r+j-1}}{P_{r-1} P_{i-1} P_{r+i} P_{j-i-1}}, E_{2}:=t^{4 i} \frac{P_{2} P_{r+i-2} P_{r+j-2}}{P_{2} P_{r-2} P_{i} P_{r+i} P_{j-i-2}} .
\end{aligned}
$$

Analogously to the previous case, we compute $K=E\left[\frac{h_{j-1} h_{j-2} h_{r+i-1} h_{r+i-2}}{h_{i-1} h_{i-2} h_{r+j-1} h_{r+j-2}}\right]$, $E_{1}=E\left[t^{2(i-1)} \frac{h_{r-1} h_{1} h_{j-i-1} h_{i-1} h_{r+j-2}}{h_{r+j-1} h_{i-2} h_{i-1} h_{r+j-2}}\right], E_{2}=E\left[t^{4 i} \frac{h_{r-2} h_{r-1} h_{j-i-2} h_{j-i-1}}{h_{r+j-2} h_{r+j-1} h_{i-2} h_{i-1}}\right]$. Hence, letting

$$
\begin{aligned}
F F(i, j, r) & :=\frac{h_{j-1} h_{j-2} h_{r+i-1} h_{r+i-2}}{h_{i-1} h_{i-2} h_{r+j-1} h_{r+j-2}}+ \\
& -t^{2(i-1)} \frac{h_{r-1} h_{1} h_{j-i-1} h_{i-1} h_{r+j-2}}{h_{r+j-1} h_{i-2} h_{i-1} h_{r+j-2}}-t^{4 i} \frac{h_{r-2} h_{r-1} h_{j-i-2} h_{j-i-1}}{h_{r+j-2} h_{r+j-1} h_{i-2} h_{i-1}}
\end{aligned}
$$

formula (11) holds if and only if $F F(i, j, r)=1$. Observe that $F F(i, j, r)$ does make sense for all integers $j \geq i \geq 2$ and $r \geq 0$. We easily check that $F F(i, j, 0)=1$. So, we now assume $r>0$ and prove that $F F(i, j, r)-F F(i, j, r-1)=0$. Arguing as in the case $k-i=2$, we let

$$
\begin{aligned}
& H_{0}:=h_{r+j-3} h_{j-1} h_{j-2} h_{r+i-1} h_{r+i-2}-h_{r+j-1} h_{j-1} h_{j-2} h_{r+i-2} h_{r+i-3} \\
& H_{1}:=t^{2(i-1)} h_{r+j-1} h_{r-2} h_{1} h_{j-i-1} h_{i-1} h_{r+j-3}+ \\
& -t^{2(i-1)} h_{r+j-3} h_{r-1} h_{1} h_{j-i-1} h_{i-1} h_{r+j-2} \\
& H_{2}:=t^{4 i} h_{r+j-1} h_{r-3} h_{r-2} h_{j-i-2} h_{j-i-1}-t^{4 i} h_{r+j-3} h_{r-2} h_{r-1} h_{j-i-2} h_{j-i-1}
\end{aligned}
$$

so that the thesis now is $H_{0}+H_{1}+H_{2}=0$.

We apply in $H_{0}$ the following replacements, in the given order: first $h_{r+i-1}=$ $t^{4} h_{r+i-3}+h_{1}$ and $h_{r+j-1}=t^{4} h_{r+j-3}+h_{1}$, then $h_{r+j-2}-h_{r+i-2}=t^{2(r+i-2)} h_{j-i-1}$. Hence, $H_{0}$ becomes

$$
\text { - } H_{0}=h_{j-1} h_{j-2} h_{r+i-2} h_{1} t^{2(r+i-2)} h_{j-i-1} \text {. }
$$


We apply in $H_{1}$ the following replacements, in the given order: first $h_{r+j-1}=$ $t^{2} h_{r+j-2}+h_{0}$ and $h_{r-1}=t^{2} h_{r-2}+h_{0}$, then $h_{r-2}-h_{r+j-2}=-t^{2(r-1)} h_{j-1}$. Hence, $H_{1}$ becomes

$-H_{1}=t^{2(i-1)} h_{r+j-3} h_{j-i-1} h_{i-1}\left(-t^{2(r-1)} h_{j-1}\right) h_{1}$.

We apply in $\mathrm{H}_{2}$ the following replacements, in the given order: first $h_{r+j-1}=$ $t^{4} h_{r+j-3}+h_{1}$ and $h_{r-1}=t^{4} h_{r-3}+h_{1}$, then $h_{r-3}-h_{r+j-3}=-t^{2(r-2)} h_{j-1}$. Hence, $\mathrm{H}_{2}$ becomes

$-H_{2}=-t^{2 i} t^{2(r+i-2)} h_{r-2} h_{j-i-2} h_{j-i-1} h_{1} h_{j-1}$.

Thus, we now have

$$
H_{0}+H_{1}+H_{2}=t^{2(r+i-2)} h_{1} h_{j-1} h_{j-i-1}\left(h_{j-2} h_{r+i-2}-h_{r+j-3} h_{i-1}-t^{2 i} h_{r-2} h_{j-i-2}\right) .
$$

Being $h_{j-2}=t^{2(j-i-1)} h_{i-1}+h_{j-i-2}$ and $h_{r+j-3}=t^{2(j-i-1)} h_{r+i-2}+h_{j-i-1}$, we finally obtain

$$
H_{0}+H_{1}+H_{2}=t^{2(r+i-2)} h_{1} h_{j-1} h_{j-i-1} h_{j-i-2}\left(h_{r+i-2}-h_{i-1}-t^{2 i} h_{r-2}\right)=0 .
$$

\section{References}

1. Abbott, J., Bigatti, A. M., and Robbiano, L.: CoCoA: a system for doing Computations in Commutative Algebra, Available at http: / / cocoa.dima.unige.it

2. Beilinson, A., Bernstein, J., Deligne, P.: Faisceaux pervers, Analysis and topology on singular spaces, I (Luminy, 1981), Astérisque, 100, Soc. Math. France, (Paris, 101982), 5-171

3. de Cataldo, M.A., Migliorini, L.: The Hodge theory of algebraic maps, Ann. Sci. École Norm. Sup. 4, 38(5), 693-750 (2005)

4. de Cataldo, M.A., Migliorini, L.: The decomposition theorem, perverse sheaves and the topology of algebraic maps, Bull. Amer. Math. Soc. (N.S.) 46, no. 4, 535-633 (2009)

5. Cheeger, J., Goresky, M., MacPherson, R.: L2-cohomology and intersection homology for singular algebraic varieties, in Seminar on Differential Geometry, volume 102 of Annals of Mathematics Studies, pages 303-340, Princeton University Press, (1982)

6. Di Gennaro, V., Franco, D.: Noether-Lefschetz. Theory with base locus, Rend. Circ. Mat. Palermo (2) 63, No. 2, 257-276, (2014). DOI 10.1007/s12215-014-0156-8

7. Di Gennaro, V., Franco, D.: On the existence of a Gysin morphism for the Blow-up of an ordinary singularity, Ann. Univ. Ferrara, Sez. VII, Sci. Mat. 63, No. 1, 75-86, (2017). https://doi.org/10.1007/ s11565-016-0253-Z

8. Di Gennaro, V., Franco, D.: Néron-Severi group of a general hypersurface, Commun. Contemp. Math., 19, No. 01, Article ID 1650004, 15 p. (2017). https://doi.org/10.1142/S0219199716500048

9. Di Gennaro, V., Franco, D.: On the topology of a resolution of isolated singularities, J. Singul. 16, 195-211 (2017). https://doi.org/10.5427/jsing.2017.16j

10. Di Gennaro, V., Franco, D.: On a Resolution of Singularities with Two Strata, Results Math. 74, no. 3, 74:115, (2019). https://doi.org/10.1007/s00025-019-1040-9

11. Di Gennaro, V., Franco, D.: On the topology of a resolution of isolated singularities, II, J. Singul. 20, 95-102 (2020). https://doi.org/10.5427/jsing.2020.20e

12. Dimca, A.: Sheaves in Topology, Springer Universitext, (2004)

13. Franco, D.: Explicit decomposition theorem for special Schubert varieties. Forum Math. 32(2), 447470 (2020)

14. Griffiths, P., Harris, J.: Principles of algebraic geometry. Pure and Applied Mathematics. WileyInterscience [John Wiley \& Sons], New York, (1978) 
15. Intersection Homology, I.I.: Goresky, M. - MacPherson, R. Invent. math. 71, 77-129 (1983)

16. Harris, J.: Algebraic Geometry. A first course, Graduate Texts in Mathematics, 133, Springer-Verlag, New York-Heidelberg, (1992)

17. Iversen, B.: Cohomology of sheaves, Springer (ISBN 3-540-16389-1; 978-3-642-82783-9/ebook) xii, 464 p. (1986)

18. Kirwan, F., Woolf, J.: An Introduction to intersection homology theory. 2nd ed., Boca Raton, FL: Chapman and Hall/CRC (ISBN 1-58488-184-4/hbk). 229 p. (2006)

19. MacPherson, R.: Global questions in the topology of singular spaces, Proceedings of the International Congress of Mathematicians, Vol.1,2 (Warsaw, 1983), 213-235

20. Manivel, L.: Symmetric functions, Schubert polynomial and degeneracy loci, SMF/AMS Texts and Monographs. 6(3). Providence, RI: American Mathematical Society (AMS). vii, 167 p. (2001)

21. Navarro Aznar, V.: Sur la théorie de Hodge des variétés algébriques à singularités isolées. Astérisque 130, 272-307 (1985)

22. Saito, M.: Mixed Hodge modules. Publ. RIMS, Kyoto Univ. 26, 221-333 (1990)

23. Voisin, C.: Hodge Theory and Complex Algebraic Geometry, I, Cambridge Studies in Advanced Mathematics 76. Cambridge University Press, (2002)

24. Williamson, G.: Hodge Theory of the Decomposition Theorem [after M.A. de Cataldo and L. Migliorini], Séminaire BOURBAKI, 2015-2016, n. 1115, pp. 31

Publisher's Note Springer Nature remains neutral with regard to jurisdictional claims in published maps and institutional affiliations. 